\title{
Characterising the Fermi-LAT BCUs: Optical Spectroscopy and Neural Networks
}

\author{
Brian van Soelen*, Johannes P Marais, Richard J Britto \\ Department of Physics, University of the Free State, 9300, Bloemfontein, South Africa \\ E-mail: vansoelenb@ufs.ac.za,maraisjp@ufs.ac.za, brittor@ufs.ac.za
}

\section{Graziano Chiaro}

Dip. Fisica \& Astronomia G. Galilei - Università di Padova, I-35131, Padova, Italy

E-mail: chiaro@pd.infn.it

\section{Lizelke Klindt, Pieter J. Meintjes}

Department of Physics, University of the Free State, 9300, Bloemfontein, South Africa

E-mail: lizelkeklindt@gmail.com, meintjpj@ufs.ac.za

\section{David Salvetti}

INAF - Istituto di Astrofisica Spaziale e Fisica Cosmica , I-20133, Milano, Italy

E-mail: salvettieiasf-milano.inaf.it

\begin{abstract}
The Fermi-LAT telescope has provided an unprecedented view of the GeV gamma-ray sky since its launch in 2008. The latest Fermi-LAT catalogue of Active Galactic Nuclei lists 1591 sources associated with AGN, of which 460 are classified as blazar candidates of uncertain type (BCU). The characterisation of the physical properties of these BCU sources is important for observational cosmology and fundamental physics, as these sources and their environments constitute a natural laboratory to study particle acceleration and matter/radiation interactions in extreme conditions. Of particular interest is the search for new and interesting/unusual sources that may be observable at very high energies by ground-based imaging atmospheric Cherenkov telescopes. Based on the observed gamma-ray properties, a number of machine learning techniques are being investigated to classify these sources. However, the classification of a blazar as a FSRQ or BL Lac depends on the optical spectral properties. Here we discuss the work that we have thus far undertaken to optically characterise a selection of sources as well as future plans to undertake classification to help calibrate an artificial neural network method.
\end{abstract}

4th Annual Conference on High Energy Astrophysics in Southern Africa

25-27 August, 2016

Cape Town, South Africa

${ }^{*}$ Speaker. 


\section{Introduction}

Blazars are radio loud Active Galactic Nuclei (AGN) where the propagation direction of the relativistic jet lies close to our line of sight. This results in the thermal component of the active galaxy being overpowered by the highly Doppler boosted non-thermal emission produced in the jet. As a result, blazars are the most numerous, non-transient, $\gamma$-ray sources detected by Fermi-LAT. In the third Fermi-LAT catalogue (3FGL), of the 1785 catalogued $\gamma$-ray sources, $1088(\sim 61 \%)$ are listed as blazars, with a further $568(\sim 32 \%)$ listed as blazar candidates [1].

Blazars are sub-divided into flat spectrum radio quasars (FSRQs) or BL Lacs based on their spectral properties. FSRQs show strong emission lines, while BL Lacs show mainly featureless spectra with weak or no emission lines. Typically, a source is classified as an FSRQ if it shows emission lines with equivalent widths $\left|W_{\lambda}\right|>5 \AA$, while it is classified as a BL Lac if the equivalent widths are $\left|W_{\lambda}\right|<5 \AA$ (e.g. [2,3]). This can be understood as FSRQs having a higher accretion rate than BL Lacs (and hence more luminous accretion discs), which leads to a greater ionization of the broad and narrow line regions (see e.g. $[4,5,6]$ and reference therein). Blazars can also be further subdivided into low-, intermediate- or high-synchrotron peaked blazars based on where the synchrotron component of the spectral energy distribution (SED) peaks. The limits are $v_{\text {peak }}<$ $10^{14} \mathrm{~Hz}, 10^{14} \mathrm{~Hz}<v_{\text {peak }}<10^{15} \mathrm{~Hz}$ and $v_{\text {peak }}>10^{15} \mathrm{~Hz}$, respectively (e.g. [7]).

The Fermi-LAT 3LAC includes 1444 extra-galactic sources (in the clean sample) which are above $|b|>10^{\circ}$ [7]. This includes 402 sources which are listed as blazar candidates of unknown type or BCUs (table 1). There are three different BCUs types, depending on whether the source has a published optical spectrum, which is not sensitive enough for classification (BCU I), whether there is no optical spectrum but the synchrotron peak in the SED can be found, (BCU II) or if there is no spectrum, but the source shows a blazar-like multi-wavelength SED (BCU III). These sources are an important sample to identify new $\mathrm{TeV}$ candidate sources that can be observed with ground-based imaging atmospheric Cherenkov telescopes (IACTs). This is particularly true for the 60 BCUs which are included in 2nd catalogue of Hard Fermi-LAT sources (2FHL) detected above $50 \mathrm{GeV}[8]$.

In order to search for new sources we are currently undertaking a project to obtain optical spectra of a selection of BCUs in order to classify the sources and determine their redshifts. The redshift is particularly important for $\mathrm{TeV}$ observations since sources beyond $z \gtrsim 1$ are unobservable at $\mathrm{TeV}$ energies due to attenuation from the extragalactic background light (EBL; [9, 10, 11]). Of particular interest is the search for non-blazar sources, since only a few of these have been detected at $\mathrm{TeV}$ energies. Currently only 6 FSRQs and 4 radio galaxies are listed in TeVCat, compared to 57 BL Lacs. ${ }^{1}$

However, the large number of sources can make detailed spectroscopic follow-up of all sources difficult. For this reason, machine learning techniques are currently been developed to classify Fermi-LAT sources based on their $\gamma$-ray properties. Recently an artificial neural network (ANN), based on the $\gamma$-ray flaring pattern was presented by Chiaro et al. [12]. However, such methods are dependent on having classified sources that can be used to train the network. Increasing the number of classifications will significantly assist with the training of these ANNs.

\footnotetext{
${ }^{1}$ http://tevcat.uchicago.edu/: accessed 22 November 2016.
} 


\begin{tabular}{lccc}
\hline AGN classification & Entire 3LAC & Clean sample & Low-latitude sample \\
\hline FSRQs & 467 & 414 & 24 \\
BL Lacs & 632 & 604 & 30 \\
BCUs & 460 & 402 & 2 \\
Total & $\mathbf{1 5 9 1}$ & $\mathbf{1 4 4 4}$ & $\mathbf{1 8 2}$ \\
\hline
\end{tabular}

Table 1: Summary of the source classification in the Fermi-LAT 3LAC [7].

This paper is structured as follows: section 2 briefly summarizes the B-FlaP method, section 3 summarizes our previous observations as well as presenting preliminary results for 3FGL J1507.63710, while the discussion and final conclusions are presented in section 4.

\section{B-FlaP method- artificial neural networks}

Chiaro et al. [12] have developed an ANN method to distinguish between FSRQ and BL Lac sources in the 3LAC catalogue based on the observed flaring pattern. The method is based on an analysis of the Empirical Cumulative Distribution Function (ECDF) of the gamma-ray light curves. These should differ in shape due to the brightness and frequency of flares observed from these sources. This classification scheme is based upon the hypothesis that BL Lacs will have fewer large flares, and smoother light curves than FSRQs.

The ANN is constructed using a two-layer feed-forward network, which consists of 10 input nodes, 6 hidden nodes and 2 output nodes. The input nodes are the flux values of the ECDFs at the $10,20,30,40,50,60,70,80,90 \& 100$ th percentiles. The ANN assigns a likelihood $\left(L_{B L L}\right)$ for a sources to be a BL Lac or an FSRQ $\left(L_{F S R Q}=1-L_{B L L}\right)$. After training the ANN on $\sim 70 \%$ of classified sources, a $90 \%$ accurate classification of the testing sample was obtained by assigning limits of $L_{B L L}>0.566$ for BL Lacs and $L_{B L L}<0.230$ for FSRQs. No classification is made for sources that are in between these limits. The ANN was applied to the Fermi-LAT BCUs and classified 342 BL Lacs and 154 FSRQs.

\section{Optical classification}

Our optical observations to classify Fermi-LAT BCUs started with sources listed in the previous Fermi-LAT 2LAC catalogue [13]. In our initial observations we focused on selecting candidate sources from the Fermi-LAT 2LAC that were unclassified, and had no redshift measurement (or had redshifts from low quality spectra) [14]. The selected candidates were limited to sources observable with South Africa based telescopes and spectroscopic observations have been undertaken with the Southern African Large Telescope (SALT; [15, 16, 17]), and the SAAO 1.9-m telescope, located at the South African Astronomical Observatory (SAAO) near Sutherland. So far 7 sources from the 2LAC catalogue have been classified as summarized in table 2 [14]. In addition, photometric observations to search for short and long term variability have been undertaken with the Sutherland High Speed Camera (SHOC; [18]) and the Watcher Robotic Telescope, respectively $[19,20]$. 


\begin{tabular}{lll}
\hline 2LAC name & Optical & ANN \\
\hline J0044.7-3702 & FSRQ & FSRQ \\
J0201.5-6626 & FSRQ & - \\
J0644.2-6713 & FSRQ & FSRQ \\
J0730.6-6607 & BL Lac & BL Lac \\
J1218.8-4827 & BL Lac & - \\
J1407.5-4257 & BL Lac & BL Lac \\
J2049.8+1001 & BL Lac & - \\
\hline
\end{tabular}

Table 2: Summary of source classification from the optical spectra [14], with a comparison to the results predicted by the ANN [12].

Our observation campaign has been extended to include unclassified sources listed in the $3 \mathrm{LAC}$ with priority given to sources included in the 2FHL catalogues. Below we briefly summarized the preliminary results for one source included it the 3LAC catalogue.

\subsection{FGL J1507.6-3710}

3FGL J1507.6-3710 is listed as a BCU II in the 3LAC catalogue, and is characterised as an intermediate synchrotron peak blazar $\left(v_{\text {peak }}=3 \times 10^{14} \mathrm{~Hz}\right)$. The source is detected at a $5.5 \sigma$ significance with an average flux of $F(1-100 \mathrm{GeV})=(4.4 \pm 0.9) \times 10^{-10} \mathrm{~cm}^{-2} \mathrm{~s}^{-1}$ with a photon index of $\Gamma=2.13 \pm 0.15$.

3FGL J1507.6-3710 was observed with the SpUpNIC spectrograph on the SAAO 1.9-m telescope on 2016 July 30 . The spectra was reduced following the standard IRAF procedures and the preliminary, normalized spectrum is shown in figure 1 . Based on the identified $\mathrm{Ca} H \& \mathrm{~K}$ and Gband this source is at a redshift of $z=0.240 \pm 0.001$. We also calculated a Ca H\&K break value of $C=\left(f_{+}-f_{-}\right) / f_{+} \sim 0.13$ where the fluxes are measured between $3750-3950 \AA$ and $4050-4250 \AA$ in the galaxy rest frame [21, 22]. Non-active galaxies have a Ca $\mathrm{H} \& \mathrm{~K}$ break which is $C \gtrsim 0.5$ [21], which indicates that the source has a non-thermal component. This, combined with the lack of strong emission lines, indicates that 3FGL J1507.6-3710 is a BL Lac. This confirms the classification predicted by the ANN [12].

\section{Discussion and conclusions}

Our on-going programme to increase the number of classified AGN sources continues to make progress. Thus far 7 new sources have been successfully classified and new observations of a selection of candidates were undertaken over three weeks in July/August 2016 with the SpUpNIC spectrograph. Here we have shown the preliminary results for one of these sources, 3FGL J1507.63710, which we have classified as a BL Lac at a redshift of $z=0.240 \pm 0.001$. Further observation programmes are planned. In addition, these results can be used to help improve the accuracy of the machine learning technique developed by Chiaro et al. [12] since a larger number of classified sources will improve the training and increase the accuracy of the ANN.

Increasing the number of classified extra-galactic sources is important to improve our understanding of the origin of extra-galactic $\gamma$-ray emission. Given the large number of extra-galactic 


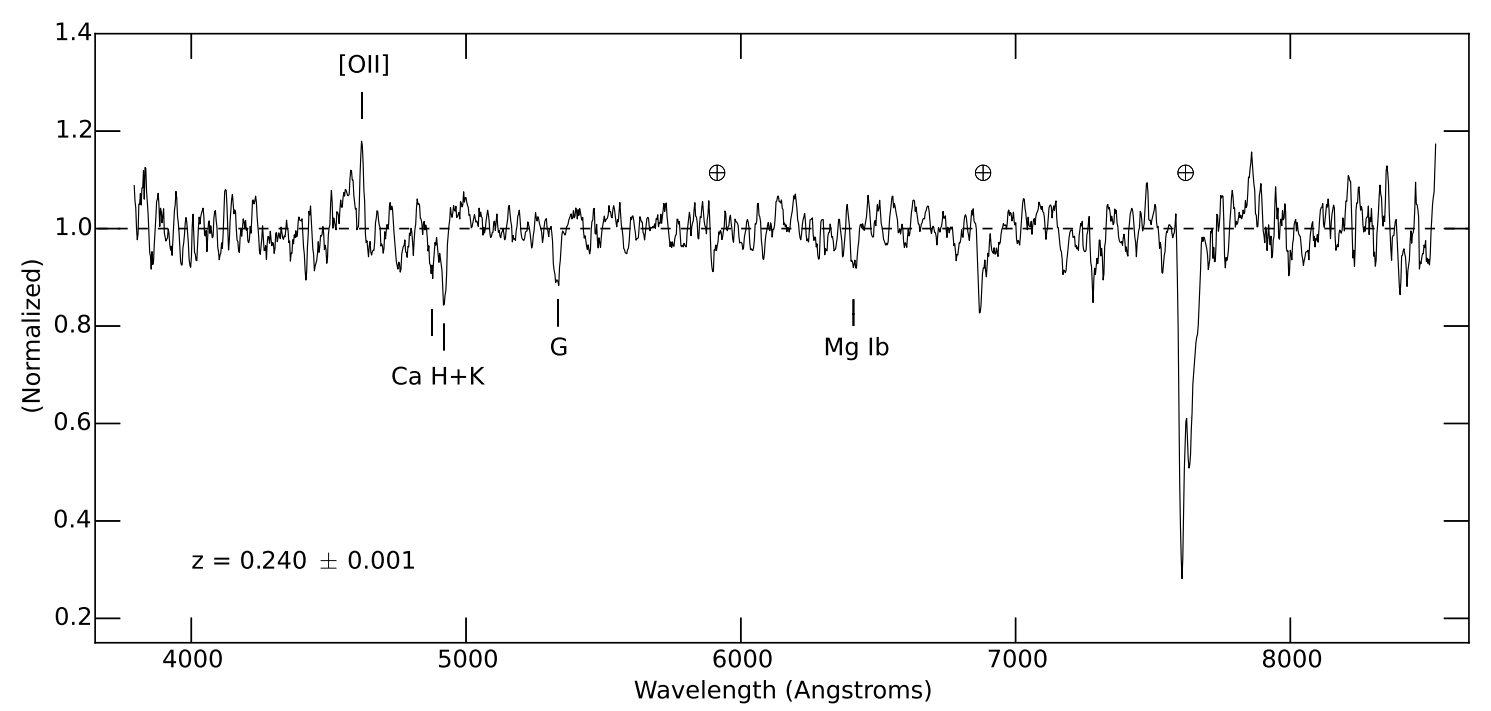

Figure 1: Combined optical spectrum for 3FGL J1507.6-3710, with the identified features marked. Telluric lines are marked with $\oplus$.

sources, and the anticipated increase in sensitivity at $\mathrm{TeV}$ energies that will be possible with the Cherenkov Telescope Array (CTA; [23]), it is important to obtain classifications for the current BCUs. Optical spectroscopic programmes and machine learning techniques, such as briefly summarized here, are important tools for undertaking such classifications.

\section{Acknowledgments}

This work is based on the research supported in part by the National Research Foundation (NRF) of South Africa for the grant No. 87919.

\section{References}

[1] F. Acero et al. (Fermi Collaboration), Fermi Large Area Telescope Third Source Catalog, ApJS 218 (2015) 23

[2] M.J.M. Marcha et al., Optical spectroscopy and polarization of a new sample of optically bright flat radio spectrum sources, MNRAS 281 (1996) 425

[3] H. Landt et al., A physical classification scheme for blazars, MNRAS 351 (2004) 83

[4] M. Böttcher and C.D. Dermer, An Evolutionary Scenario for Blazar Unification, ApJ 564 (2002) 86

[5] A. Cavaliere and V. D’Elia, The Blazar Main Sequence, ApJ 571 (2002) 226

[6] G. Ghisellini et al., General physical properties of bright Fermi blazars, MNRAS 402 (2010) 497

[7] M. Ackermann et al. (Fermi Collaboration), The Third Catalog of Active Galactic Nuclei Detected by the Fermi Large Area Telescope, ApJ 810 (2015) 14

[8] M. Ackermann et al. (Fermi Collaboration), 2FHL: The Second Catalog of Hard Fermi-LAT Sources, ApJS 222 (2016) 5 
[9] R.J. Gould and G. Schréder, Opacity of the Universe to High-Energy Photons, Physical Review Letters 16 (1966) 252

[10] M.G. Hauser and E. Dwek, The Cosmic Infrared Background: Measurements and Implications, ARA\&A 39 (2001) 249

[11] F. Aharonian et al. (H.E.S.S. Collaboration), A low level of extragalactic background light as revealed by $\gamma$-rays from blazars, Nature $\mathbf{4 4 0} 1018$

[12] G. Chiaro et al. Blazar flaring patterns (B-FlaP) classifying blazar candidate of uncertain type in the third Fermi-LAT catalogue by artificial neural networks, MNRAS 462 (2016) 3180

[13] M. Ackermann et al. (Fermi Collaboration), The Second Catalog of Active Galactic Nuclei Detected by the Fermi Large Area Telescope, ApJ 743 (2011) 171

[14] L. Klindt et al., Optical spectroscopic classification of a selection of Southern Hemisphere Fermi-LAT unclassified blazars, MNRAS 467 (2017) 2537

[15] D.A.H. Buckley et al., Completion and commissioning of the Southern African Large Telescope, SPIE 6267 (2006) 32

[16] E.B. Burgh et al., Prime Focus Imaging Spectrograph for the Southern African Large Telescope: optical design, SPIE 4841 (2003) 1463

[17] H.A. Kobulnicky et al., Prime focus imaging spectrograph for the Southern African large telescope: operational modes, SPIE 4841 (2003) 1634

[18] R. Coppejans et al., Characterizing and Commissioning the Sutherland High-Speed Optical Cameras (SHOC), PASP 125 (2013) 976

[19] L. Klindt et al., Optical variability of unidentified Active Galactic Nuclei with blazar characteristics in the Fermi-2LAC catalogue in proceedings of 3rd Annual Conference on High Energy Astrophysics in Southern Africa, POS (HEASA2 015$) 008$ (2015)

[20] B. van Soelen et al., Optical observations of Very High Energy Sources from the Boyden Observatory in proceedings of 3rd Annual Conference on High Energy Astrophysics in Southern Africa, POS (HEASA2015) 007 (2015)

[21] A. Dressler and S.A. Shectman, Systematics of the 4000 Angström break in the spectra of galaxies, AJ 94 (1987) 899

[22] H. Landt et al., The classification of BL Lacertae objects: the Ca H\&K break, MNRAS 336 (2002) 945

[23] M Actis et al. (CTA Consortium), Design Concepts for the Cherenkov Telescope Array, Experimental Astronomy 32 (2011) 193 